\title{
An Analytical Model for Deriving the 3-D Potentials and the Front and Back Gate Threshold Voltages of a Mesa- Isolated Small Geometry Fully Depleted SOI MOSFET
}

\author{
Jae Bin Lee and Chung Ha Suh
}

\begin{abstract}
For a mesa-isolated small geometry SOI MOSFET, the potentials in the silicon film, front, back, and side-wall oxide layers can be derived threedimensionally. Using Taylor's series expansions of the trigonometric functions, the derived potentials are written in terms of the natural length that can be determined by using the derived formula. From the derived 3-D potentials, the minimum values of the front and the back surface potentials are derived and used to obtain the closed-form expressions for the front and back gate threshold voltages as functions of various device parameters and applied bias voltages. Obtained results can be found to explain the draininduced threshold voltage roll-off and the narrow width effect of a fully depleted small geometry SOI MOSFET in a unified manner.
\end{abstract}

Index Terms-3-D analytical SOI MOSFET model, threshold voltage roll-off, short channel effect, narrow width effect, natural length

\section{INTRODUCTION}

Since silicon-on-insulator (SOI) MOSFETs are inherently possessed of several superior electrical characteristics over bulk-type MOSFETs, such as better isolation between source (drain) and substrate, punch through suppression, enhanced current driving capability,

Manuscript received May. 31, 2012; revised Aug. 19, 2012. School of Electronic \& Electrical Eng., Hongik University, Korea E-mail : chsuh@hongik.ac.kr and suppressed short channel effects [1-6], they are nowadays facilitating the integration level of VLSI up to ULSI. In order to achieve extremely high integration in ULSI, the geometry of a SOI MOSFET is very small by reducing the channel (gate) width as well as the channel length. Then for a mesa-isolated small geometry fully depleted (FD) SOI MOSFET, the potential in the silicon film (body) becomes strongly coupled with the potentials in the front, back, and side-wall oxide layers. Thus, in order to have accurate and rigorous modeling the characteristics of a mesa-isolated small geometry SOI MOSFET, three-dimensional analysis should be required. Especially, the narrow width effect and the short channel effect will offer combined influence on the threshold voltage. The threshold voltage is very crucial parameter since it can estimate several features of a MOSFET such as channel conductance, drain saturation voltage/current, turn-on/off speed, etc. Recently, by extending the twodimensional (2-D) analytical models [7-13] up to threedimensional (3-D) analysis, 3-D models [14-17] for the threshold voltage have been suggested. However, employed 2-D analytical models were somewhat oversimplified in the sense that the potentials in both front and back oxide layers were derived by using the gradual channel approximation (GCA), the potential in the silicon body was derived by decomposing it into a 1D Poisson's equation and a 2-D Laplace's equation. In solving the 2-D Laplace's equation, rather complicated infinite series expansions have been required to satisfy the suggested equi-potential boundary conditions at both planes of source-body and drain-body $n^{+}-p$ junctions. Hence, the front and back surface potentials have been 
expressed as certain infinite series expansions, which have been approximated by taking only one term in the derivation of the minimum front and back surface potentials. These approximations seem to be somewhat oversimplified and inconsistent with the suggested equipotential boundary conditions. In the 3-D models [14, 17], complicated double summations of infinite series expansions have been used for deriving the 3-D potential in the silicon body. However, since the approximation of the double summations of infinite series by taking only one term seems to be rather oversimplified and the analytical logic of the above 3-D models will be collapsed in case that one of oxide layers is fairly thick, the derived expressions for the front and back gate threshold voltages seem to be more or less ambiguous.

In this paper, starting from the potential expressions obtained by using the GCA and updating them iteratively by taking into account their $y$ - and $z$-variations, we can derive the potentials in the silicon body, front, back, and side-wall oxide layers three-dimensionally. From the derived 3-D potentials, the minimum values of the front and back surface potentials can be derived and used to obtain the closed-form expressions for the front and back gate threshold voltages as functions of various device parameters and applied bias voltages.

This paper is organized as follows. In Section II, the basic model formulation is described. In Section III, some computation results and discussion are contained. A summary and a conclusion are in Section IV.

\section{Model Formulation}

Schematic cross section views of a mesa-isolated fully depleted SOI MOSFET are shown in Figs. 1(a) and 1(b). Here, $x$ is the channel-depth directed coordinate from the front oxide/silicon interface toward the silicon body; $y$ is the longitudinal coordinate from the source side end toward the drain; $z$ is the transversal coordinate from the middle of the silicon film toward one side end; $L$ and $w$ are the channel length and width, respectively; $t_{s i}$ is the silicon film thickness; $t_{o f}, t_{o b}$, and $t_{o w}$ are the thicknesses of the front, back, and sidewall oxide layers, respectively; $V_{G S}, V_{B S}$, and $V_{D S}$ are front gate, back gate, and drain voltages, respectively. In order to establish an accurate and rigorous model for the front and back threshold voltages of a mesa-isolated

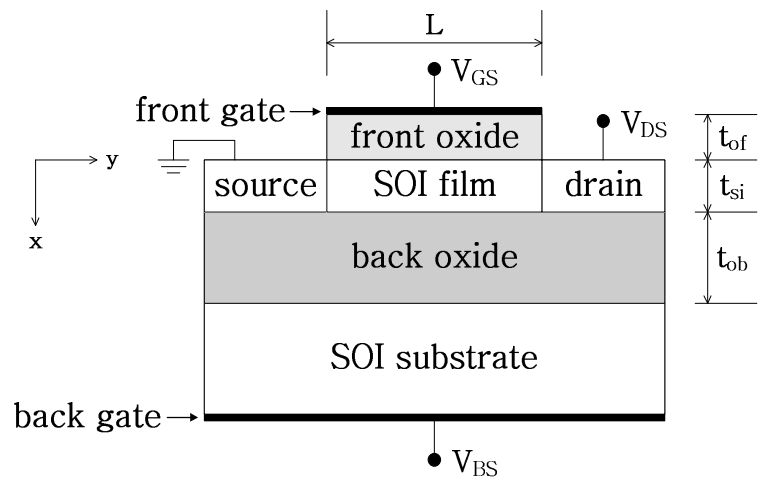

(a)

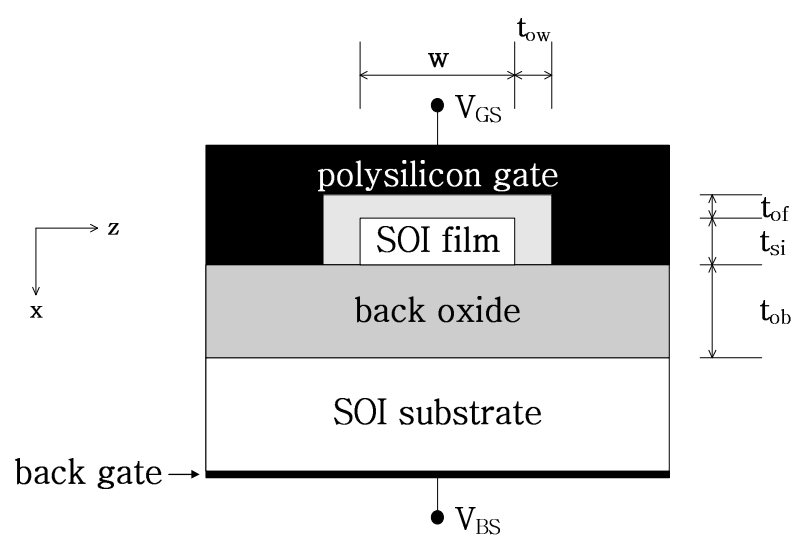

(b)

Fig. 1. (a), (b) Schematic cross-section views of a mesaisolated n-channel SOI MOSFET to be modeled.

small geometry SOI MOSFET, let us assume that the device structure is symmetric about the axis of $z=0$, the space charge densities in the oxide layers are negligible, and the silicon body is uniformly p-doped and fairly thin so that the silicon body is fully depleted in the sub-threshold regime. Then we can consider the following 3-D Laplace/Poisson's equation:

$$
\frac{\partial^{2} \psi}{\partial x^{2}}+\frac{\partial^{2} \psi}{\partial y^{2}}+\frac{\partial^{2} \psi}{\partial z^{2}}= \begin{cases}0, & \text { in the oxide layers, } \\ \frac{q N_{A}}{\varepsilon_{s i}}, & \text { in the silicon body }\end{cases}
$$

where $\psi(x, y, z)$ is the 3 -D electrostatic potential, $q$ is the elementary charge, $N_{A}$ is the uniform acceptor density in the silicon body, and $\varepsilon_{s i}$ is the dielectric constant of silicon. For convenience, we separately denote the potentials in the silicon film, front, back, and side-wall oxide layers as $\psi^{s i}(x, y, z), \quad \psi^{o f}(x, y, z)$, $\psi^{o b}(x, y, z)$, and $\psi^{o w}(x, y, z)$, respectively. Boundary conditions subject to Eq. (1) will be as follows: 


$$
\begin{gathered}
\psi^{o f}\left(-t_{o f}, y, z\right)=V_{G S}-\phi_{m s f}=V_{G S}^{\prime}, \\
\psi^{o b}\left(t_{s i}+t_{o b}, y, z\right)=V_{B S}-\phi_{m s b}=V_{B S}^{\prime}, \\
\psi^{o w}\left(x, y, \pm w / 2 \pm t_{o w}\right)=V_{G S}-\phi_{m s f}=V_{G S}^{\prime}, \\
\psi^{s i}(0, y, z)=\psi^{o f}(0, y, z)=\psi_{s}(y, z), \\
\psi^{s i}\left(t_{s i}, y, z\right)=\psi^{o b}\left(t_{s i}, y, z\right)=\psi_{b}(y, z), \\
\psi^{s i}(x, y, \pm w / 2)=\psi^{o w}(x, y, \pm w / 2), \\
\left.\frac{\partial \psi^{s i}}{\partial x}\right|_{x=0}=\frac{\varepsilon_{o x}}{\varepsilon_{s i}}\left(\left.\frac{\partial \psi^{o f}}{\partial x}\right|_{x=0}-\frac{Q_{s s f}}{\varepsilon_{o x}}\right), \\
\left.\frac{\partial \psi^{s i}}{\partial x}\right|_{x=t_{s i}}=\frac{\varepsilon_{o x}}{\varepsilon_{s i}}\left(\left.\frac{\partial \psi^{o b}}{\partial x}\right|_{z= \pm t_{s i}}+\frac{Q_{s s b}}{\varepsilon_{o x}}\right), \\
=\frac{\varepsilon_{o x}}{\varepsilon_{s i}}\left(\left.\frac{\partial \psi^{o w}}{\partial z}\right|_{z= \pm w / 2} \pm \frac{Q_{s s w}}{\varepsilon_{o x}}\right),
\end{gathered}
$$

where $\phi_{m s f}=\phi_{m f}-\phi_{s} \quad\left(\phi_{m s b}=\phi_{m b}-\phi_{s}\right)$ is the difference between the work functions $\phi_{m f}\left(\phi_{m b}\right)$ for the front (back) gate electrode and $\phi_{s}$ for the silicon body; $\psi_{s}(y, z)$ and $\psi_{b}(y, z)$ are the front and back surface potentials, respectively; $\varepsilon_{o x}$ is the dielectric constant of oxide; and $Q_{s f}\left(Q_{s s b}, Q_{s s w}\right)$ is the surface state charge areal density at the front (back, side-wall) oxide/silicon interface. Starting from the potentials obtained by neglecting both $\partial^{2} \psi_{s}(y, z) / \partial y^{2}+\partial^{2} \psi_{s}(y, z) / \partial z^{2}$ and $\partial^{2} \psi_{b}(y, z) / \partial y^{2}+\partial^{2} \psi_{b}(y, z) / \partial z^{2}$, and updating the potential expressions iteratively by taking into account their $y$ - and $z$-variations, we can write $\psi^{\circ f}(x, y, z)$, $\psi^{o b}(x, y, z)$, and $\psi^{s i}(x, y, z)$ in the 3 -D expressions:

$$
\begin{aligned}
& \psi^{o f}(x, y, z)=V_{G S}^{\prime}+\frac{x+t_{o f}}{t_{o f}}\left[\psi_{s}(y, z)-V_{G S}^{\prime}\right] \\
& -\frac{1}{3 ! t_{o f}}\left[\left(x+t_{o f}\right)^{3}-t_{o f}^{2}\left(x+t_{o f}\right)\right] \Delta_{y z} \psi_{s}(y, z) \\
& +\frac{1}{5 ! t_{o f}}\left[\left(x+t_{o f}\right)^{5}-\frac{10}{3} t_{o f}^{2}\left(x+t_{o f}\right)^{3}+\frac{7}{3} t_{o f}^{4}\left(x+t_{o f}\right)\right] \Delta_{y z}^{2} \psi_{s}(y, z) \\
& -\cdots, \\
& \psi^{o b}(x, y, z)=V_{B S}^{\prime}-\frac{x^{\prime}-t_{o b}}{t_{o b}}\left[\psi_{b}(y, z)-V_{B S}^{\prime}\right] \\
& +\frac{1}{3 ! t_{o b}}\left[\left(x^{\prime}-t_{o b}\right)^{3}-t_{o b}^{2}\left(x^{\prime}-t_{o b}\right)\right] \Delta_{y z} \psi_{b}(y, z) \\
& -\frac{1}{5 ! t_{o b}}\left[\left(x^{\prime}-t_{o b}\right)^{5}-\frac{10}{3} t_{o b}^{2}\left(x^{\prime}-t_{o b}\right)^{3}+\frac{7}{3} t_{o b}^{4}\left(x^{\prime}-t_{o b}\right)\right] \Delta_{y z}^{2} \psi_{b}(y, z) \\
& +\cdots, \\
& \psi^{i i}(x, y, z)=\frac{q N_{A}}{2 \varepsilon_{s i}} x x^{\prime}-\frac{x^{\prime}}{t_{s i}} \psi_{s}(y, z)+\frac{x}{t_{s i}} \psi_{b}(y, z)
\end{aligned}
$$

$$
\begin{aligned}
& +\frac{1}{3 ! t_{s i}}\left(x^{3}-t_{s i}^{2} x^{\prime}\right) \Delta_{y z} \psi_{s}(y, z)-\frac{1}{3 ! t_{s i}}\left(x^{3}-t_{s i}^{2} x\right) \Delta_{y z} \psi_{b}(y, z) \\
& -\frac{1}{5 ! t_{s i}}\left(x^{\prime}-\frac{10}{3} t_{s i}^{2} x^{\prime}+\frac{7}{3} t_{s i}^{4} x^{\prime}\right) \Delta_{y z}^{2} \psi_{s}(y, z) \\
& +\frac{1}{5 ! t_{s i}}\left(x^{5}-\frac{10}{3} t_{s i}^{2} x^{3}+\frac{7}{3} t_{s i}^{4} x\right) \Delta_{y z}^{2} \psi_{b}(y, z)+\cdots,
\end{aligned}
$$

where $x^{\prime}=x-t_{s i}, \quad \Delta_{y z}=\partial^{2} / \partial y^{2}+\partial^{2} / \partial z^{2}, \quad$ and $\quad \Delta_{y z}^{2}=$ $\left(\partial^{2} / \partial y^{2}+\partial^{2} / \partial z^{2}\right)^{2}$. Using Eqs. (5), (6), and (7), we can rewrite Eqs. (4a) and (4b) as:

$$
\begin{aligned}
& \left(\frac{1}{t_{s i}}+\frac{\eta}{t_{o f}}\right) \psi_{s}(y, z)-\frac{1}{t_{s i}} \psi_{b}(y, z) \\
& -\left(\frac{t_{s i}}{3}+\frac{\eta t_{o f}}{3}\right) \Delta_{y z} \psi_{s}(y, z)-\frac{t_{s i}}{6} \Delta_{y z} \psi_{b}(y, z) \\
& -\left(\frac{t_{s i}^{3}}{45}+\frac{\eta t_{o f}^{3}}{45}\right) \Delta_{y z}^{2} \psi_{s}(y, z)-\frac{7 t_{s i}^{3}}{360} \Delta_{y z}^{2} \psi_{b}(y, z)-\cdots \\
& =\frac{\eta}{t_{o f}}\left(V_{G S}-V_{F B f}-\frac{q N_{A} t_{o f} t_{s i}}{2 \varepsilon_{o x}}\right), \\
& \left(\frac{1}{t_{s i}}+\frac{\eta}{t_{o b}}\right) \psi_{b}(y, z)-\frac{1}{t_{s i}} \psi_{s}(y, z) \\
& -\left(\frac{t_{s i}}{3}+\frac{\eta t_{o b}}{3}\right) \Delta_{y z} \psi_{b}(y, z)-\frac{t_{s i}}{6} \Delta_{y z} \psi_{s}(y, z) \\
& -\left(\frac{t_{s i}^{3}}{45}+\frac{\eta t_{o b}^{3}}{45}\right) \Delta_{y z}^{2} \psi_{b}(y, z)-\frac{7 t_{s i}^{3}}{360} \Delta_{y z}^{2} \psi_{s}(y, z)-\cdots \\
& =\frac{\eta}{t_{o b}}\left(V_{B S}-V_{F B b}-\frac{q N_{A} t_{o b} t_{s i}}{2 \varepsilon_{o x}}\right),
\end{aligned}
$$

where $\eta=\varepsilon_{o x} / \varepsilon_{s i}, \quad V_{F B f}=\phi_{m s f}-t_{o f} Q_{s f} / \varepsilon_{o x}$, and $V_{F B b}=$ $\phi_{m s b}-t_{o b} Q_{s s b} / \varepsilon_{o x}$. For the conditions that Eqs. (8) and (9) satisfy simultaneously, we may suggest that

$$
\begin{aligned}
& \left(\frac{\partial^{2}}{\partial y^{2}}+\frac{\partial^{2}}{\partial z^{2}}\right) \psi_{s}(y, z)=\frac{1}{\lambda_{x}^{2}}\left[\psi_{s}(y, z)-\Phi_{s}\right], \\
& \left(\frac{\partial^{2}}{\partial y^{2}}+\frac{\partial^{2}}{\partial z^{2}}\right) \psi_{b}(y, z)=\frac{1}{\lambda_{x}^{2}}\left[\psi_{b}(y, z)-\Phi_{b}\right] .
\end{aligned}
$$

Then we can rewrite Eqs. (8) and (9) in the forms:

$$
\begin{aligned}
& \frac{1}{\lambda_{x}}\left[\cot \left(\frac{t_{s i}}{\lambda_{x}}\right)+\eta \cot \left(\frac{t_{o f}}{\lambda_{x}}\right)\right]\left[\psi_{s}(y, z)-\Phi_{s}\right] \\
& -\frac{1}{\lambda_{x}} \csc \left(\frac{t_{s i}}{\lambda_{x}}\right)\left[\psi_{b}(y, z)-\Phi_{b}\right]
\end{aligned}
$$


$=\frac{\eta}{t_{o f}}\left(V_{G S}-V_{F B f}-\frac{q N_{A} t_{o f} t_{s i}}{2 \varepsilon_{o x}}\right)-\left(\frac{1}{t_{s i}}+\frac{\eta}{t_{o f}}\right) \Phi_{s}+\frac{1}{t_{s i}} \Phi_{b}$,

$\frac{1}{\lambda_{x}}\left[\cot \left(\frac{t_{s i}}{\lambda_{x}}\right)+\eta \cot \left(\frac{t_{o b}}{\lambda_{x}}\right)\right]\left[\psi_{b}(y, z)-\Phi_{b}\right]$

$-\frac{1}{\lambda_{x}} \csc \left(\frac{t_{s i}}{\lambda_{x}}\right)\left[\psi_{s}(y, z)-\Phi_{s}\right]$

$=\frac{\eta}{t_{o b}}\left(V_{B S}-V_{F B b}-\frac{q N_{A} t_{o b} t_{s i}}{2 \varepsilon_{o x}}\right)-\left(\frac{1}{t_{s i}}+\frac{\eta}{t_{o b}}\right) \Phi_{b}+\frac{1}{t_{s i}} \Phi_{s}$,

where we have replaced $1 / \theta-\theta / 3-\theta^{3} / 45-\cdots$ and $1 / \theta+\theta / 6+7 \theta^{3} / 360+\cdots \quad$ with $\cot \theta$ and $\csc \theta$, respectively. Combining Eqs. (12) and (13), we can get

$$
\begin{aligned}
\Phi_{s}= & \frac{\eta t_{s i}+t_{o b}}{\eta t_{s i}+t_{o f}+t_{o b}}\left(V_{G S}-V_{F B f}-\frac{q N_{A} t_{o f} t_{s i}}{2 \varepsilon_{o x}}\right) \\
& +\frac{t_{o f}}{\eta t_{s i}+t_{o f}+t_{o b}}\left(V_{B S}-V_{F B b}-\frac{q N_{A} t_{o b} t_{s i}}{2 \varepsilon_{o x}}\right), \\
\Phi_{b}= & \frac{\eta t_{s i}+t_{o f}}{\eta t_{s i}+t_{o f}+t_{o b}}\left(V_{B S}-V_{F B b}-\frac{q N_{A} t_{o b} t_{s i}}{2 \varepsilon_{o x}}\right) \\
& +\frac{t_{o b}}{\eta t_{s i}+t_{o f}+t_{o b}}\left(V_{G S}-V_{F B f}-\frac{q N_{A} t_{o f} t_{s i}}{2 \varepsilon_{o x}}\right), \\
& \cot \left(\frac{t_{s i}}{\lambda_{x}}\right)=\frac{\frac{1}{\eta}-\eta \cot \left(\frac{t_{o f}}{\lambda_{x}}\right) \cot \left(\frac{t_{o b}}{\lambda_{x}}\right)}{\cot \left(\frac{t_{o f}}{\lambda_{x}}\right)+\cot \left(\frac{t_{o b}}{\lambda_{x}}\right)} .
\end{aligned}
$$

Since $1 / \theta-\theta / 3-\theta^{3} / 45-\cdots=\cot \theta$ is valid in the range of $|\theta|<\pi$, the root of Eq. (16) will be derived uniquely in the range of $\lambda_{x}>\pi^{-1} \max \left\{t_{s i}, t_{o f}, t_{o b}\right\}$. For a symmetric dual gate (SDG) MOSFET ( $t_{o f}=t_{o b}=t_{o x}$, $V_{F B f}=V_{F B b}=V_{F B}, \quad V_{G S}=V_{B S}$ ), we can have

$$
\begin{gathered}
\Phi_{s}=\Phi_{b}=V_{G S}-V_{F B}-\frac{q N_{A} t_{o x} t_{s i}}{2 \varepsilon_{o x}}, \\
\tan \left(\frac{t_{s i}}{2 \lambda_{x}}\right)=\eta \cot \left(\frac{t_{o x}}{\lambda_{x}}\right) .
\end{gathered}
$$

In case that $t_{o f}<<\lambda_{x}$ and $t_{o b}<<\lambda_{x}$, Eq. (16) can be simplified as

$$
\tan \left(\frac{t_{s i}}{\lambda_{x}}\right)=\frac{\frac{1}{\lambda_{x}}\left(\frac{t_{o f}}{\eta}+\frac{t_{o b}}{\eta}\right)}{\frac{t_{o f} t_{o b}}{\eta^{2} \lambda_{x}^{2}}-1},
$$

which was used in $[14,17]$. Since most of mathematics used in $[14,17]$ are strictly based on Eq. (19), the models $[14,17]$ will be very restricted 3-D models. Furthermore, in $[14,17], \psi^{s i}(x, y, z)$ has been expressed as a variable dependent on $x$ in the sinusoidal fashion and on $z$ in the hyperbolic fashion. This expression would not be adequate when considering the fact that $x$ and $z$ are exchangeable, especially in the case of $t_{o f}=t_{o b \text { : }}$.

Meanwhile, considering that $\partial \psi_{s}(y, z) /\left.\partial z\right|_{z=0}=0$ and $\partial \psi_{b}(y, z) /\left.\partial z\right|_{z=0}=0$, and also considering that the $z$-dependency of $\psi^{s i}(x, y, z)$ will be very similar to the $x$ - dependency, we may solve Eqs. (10) and (11), respectively, in the forms:

$$
\begin{aligned}
& \psi_{s}(y, z)=\Phi_{s}+\left[\psi_{s c}(y)-\Phi_{s}\right] \cos \left(\frac{z}{\lambda_{z}}\right), \\
& \psi_{b}(y, z)=\Phi_{b}+\left[\psi_{b c}(y)-\Phi_{b}\right] \cos \left(\frac{z}{\lambda_{z}}\right),
\end{aligned}
$$

where

$$
\begin{aligned}
& \psi_{s c}(y)=\psi_{s}(y, 0)=\Phi_{s} \\
& +\operatorname{csch}\left(\frac{L}{\lambda}\right)\left\{\left[\psi_{s c}(0)-\Phi_{s}\right] \sinh \left(\frac{L-y}{\lambda}\right)+\left[\psi_{s c}(L)-\Phi_{s}\right] \sinh \left(\frac{y}{\lambda}\right)\right\}, \\
& \psi_{b c}(y)=\psi_{b}(y, 0)=\Phi_{b} \\
& +\operatorname{csch}\left(\frac{L}{\lambda}\right)\left\{\left[\psi_{b c}(0)-\Phi_{b}\right] \sinh \left(\frac{L-y}{\lambda}\right)+\left[\psi_{b c}(L)-\Phi_{b}\right] \sinh \left(\frac{y}{\lambda}\right)\right\},
\end{aligned}
$$

with

$$
\lambda=\frac{1}{\sqrt{\lambda_{x}^{-2}+\lambda_{z}^{-2}}} .
$$

Then we can rewrite Eqs. (5), (6), and (7) in the forms:

$$
\begin{aligned}
& \psi^{o f}(x, y, z)=V_{G S}^{\prime}+\frac{x+t_{o f}}{t_{o f}}\left(\Phi_{s}-V_{G S}^{\prime}\right) \\
& +\csc \left(\frac{t_{o f}}{\lambda_{x}}\right) \sin \left(\frac{x+t_{o f}}{\lambda_{x}}\right)\left[\psi_{s c}(y)-\Phi_{s}\right] \cos \left(\frac{z}{\lambda_{z}}\right), \\
& \psi^{o b}(x, y, z)=V_{B S}^{\prime}-\frac{x^{\prime}-t_{o b}}{t_{o b}}\left(\Phi_{b}-V_{B S}^{\prime}\right) \\
& -\csc \left(\frac{t_{o b}}{\lambda_{x}}\right) \sin \left(\frac{x^{\prime}-t_{o b}}{\lambda_{x}}\right)\left[\psi_{b c}(y)-\Phi_{b}\right] \cos \left(\frac{z}{\lambda_{z}}\right),
\end{aligned}
$$




$$
\begin{aligned}
& \psi^{s i}(x, y, z)=\frac{q N_{A}}{2 \varepsilon_{s i}} x x^{\prime}-\frac{x^{\prime}}{t_{s i}} \Phi_{s}+\frac{x}{t_{s i}} \Phi_{b} \\
& -\csc \left(\frac{t_{s i}}{\lambda_{x}}\right) \sin \left(\frac{x^{\prime}}{\lambda_{x}}\right)\left[\psi_{s c}(y)-\Phi_{s}\right] \cos \left(\frac{z}{\lambda_{z}}\right) \\
& +\csc \left(\frac{t_{s i}}{\lambda_{x}}\right) \sin \left(\frac{x}{\lambda_{x}}\right)\left[\psi_{b c}(y)-\Phi_{b}\right] \cos \left(\frac{z}{\lambda_{z}}\right) .
\end{aligned}
$$

On the other hand, we may suggest $\psi^{o w}(x, y, z)$ of the form:

$$
\begin{gathered}
\psi^{o w}(x, y, z)=V_{G S}^{\prime}-\frac{z^{\prime}}{t_{o w}}\left(\frac{q N_{A}}{2 \varepsilon_{s i}} x x^{\prime}-\frac{x^{\prime}}{t_{s i}} \Phi_{s}+\frac{x}{t_{s i}} \Phi_{b}-V_{G S}^{\prime}\right) \\
+\frac{q N_{A}}{\varepsilon_{s i}} \frac{1}{3 ! t_{o w}}\left(z^{\prime 3}-t_{o w}^{2} z^{\prime}\right)-\csc \left(\frac{t_{o w}}{\lambda_{z}}\right) \sin \left(\frac{z^{\prime}}{\lambda_{z}}\right) \varphi_{w}(x, y),
\end{gathered}
$$

where $z^{\prime}=z \mp\left(w / 2+t_{\text {ow }}\right)$ and

$$
\begin{aligned}
\varphi_{w}(x, y) & =-\csc \left(\frac{t_{s i}}{\lambda_{x}}\right) \sin \left(\frac{x^{\prime}}{\lambda_{x}}\right)\left[\psi_{s c}(y)-\Phi_{s}\right] \cos \left(\frac{w}{2 \lambda_{z}}\right) \\
& +\csc \left(\frac{t_{s i}}{\lambda_{x}}\right) \sin \left(\frac{x}{\lambda_{x}}\right)\left[\psi_{b c}(y)-\Phi_{b}\right] \cos \left(\frac{w}{2 \lambda_{z}}\right) .
\end{aligned}
$$

Using Eqs. (27) and (28) into Eq. (4c), we may have

$$
\begin{aligned}
& \frac{1}{\lambda_{z}}\left[\tan \left(\frac{w}{2 \lambda_{z}}\right)-\eta \cot \left(\frac{t_{o w}}{\lambda_{z}}\right)\right] \varphi_{w}(x, y) \\
& =\frac{\eta}{t_{o w}}\left(\frac{q N_{A}}{2 \varepsilon_{s i}} x x^{\prime}-\frac{x^{\prime}}{t_{s i}} \Phi_{s}+\frac{x}{t_{s i}} \Phi_{b}-V_{G S}+V_{F B w}-\frac{q N_{A}}{3 \varepsilon_{s i}} t_{o w}^{2}\right),
\end{aligned}
$$

where $V_{F B w}=\phi_{m s f}-t_{o w} Q_{s s w} / \varepsilon_{o x}$. Considering that the electrostatic corner effects, we may assume that $V_{F B w}$ is varied with $x$ in order to satisfy that

$$
\begin{aligned}
& V_{F B w}(x) \\
& =\frac{q N_{A}}{3 \varepsilon_{s i}} t_{o w}^{2}-\left(\frac{q N_{A}}{2 \varepsilon_{s i}} x x^{\prime}-\frac{x^{\prime}}{t_{s i}} \Phi_{s}+\frac{x}{t_{s i}} \Phi_{b}-V_{G S}\right) .
\end{aligned}
$$

Then we have

$$
\tan \left(\frac{w}{2 \lambda_{z}}\right)=\eta \cot \left(\frac{t_{o w}}{\lambda_{z}}\right)
$$

Considering that $\theta+\theta^{3} / 3+2 \theta^{5} / 15+\cdots=\tan (\theta)$ and $1 /(2 \theta)-2 \theta / 3-(2 \theta)^{3} / 45-\cdots=\cot (2 \theta)$ can be valid in the range of $|\theta|<\pi / 2, \quad \lambda_{z}$ will be derived uniquely in the range of $\lambda_{z}>\pi^{-1} \max \left\{w, t_{o w}\right\}$. From Eq. (32), we get

$$
\frac{\partial \lambda_{z}}{\partial w}=\frac{\lambda_{z}}{w+2 t_{o w}\left[\eta+\left(\frac{1}{\eta}-\eta\right) \sin ^{2}\left(\frac{w}{2 \lambda_{z}}\right)\right]} .
$$

Using Eqs. (22) and (23) and performing the similar algebraic procedure given in $[18,19]$, the minimum values of $\psi_{s}(y, 0)$ and $\psi_{b}(y, 0)$, i.e., $\psi_{s, \min }$ and $\psi_{b, \min }$ can be derived as

$$
\begin{aligned}
& \psi_{s, \text { min }}=\Phi_{s} \\
& +\left[\psi_{s c}(0)-\Phi_{s}\right] \sqrt{1-\left[\tanh \left(\frac{L}{2 \lambda}\right)-\frac{\psi_{s c}(L)-\psi_{s c}(0)}{\psi_{s c}(0)-\Phi_{s}} \operatorname{csch}\left(\frac{L}{\lambda}\right)\right]^{2}}, \\
& \psi_{b, \text { min }}=\Phi_{b} \\
& +\left[\psi_{b c}(0)-\Phi_{b}\right] \sqrt{1-\left[\tanh \left(\frac{L}{2 \lambda}\right)-\frac{\psi_{b c}(L)-\psi_{b c}(0)}{\psi_{b c}(0)-\Phi_{b}} \operatorname{csch}\left(\frac{L}{\lambda}\right)\right]^{2}} .
\end{aligned}
$$

In the sub-threshold regime, ohmic drops due to the parasitic source and drain resistances can be negligible. Thus, we may write $\psi_{s c}(0) \approx \psi_{b c}(0) \approx V_{b i}$ and $\psi_{s c}(L)-\psi_{s c}(0) \approx \psi_{b c}(L)-\psi_{b c}(0) \approx V_{D S}$, where $V_{b i}$ is the built-in voltage across the $n^{+}-p$ source (drain)/ body junction. Let us suggest that the sub-threshold conditions of the front and back surface channels, i.e., $V_{G S} \leq V_{T f}$ and $V_{B S} \leq V_{T b}$, are equivalent to $\psi_{s, \text { min }} \leq 2 \Phi_{B}$ and $\psi_{b, \text { min }} \leq 2 \Phi_{B}$, where $V_{T f}$ and $V_{T b}$ are the front and back gate threshold voltages, respectively, and $\Phi_{B}=\left(k_{B} T / q\right) \ln \left(N_{A} / n_{i}\right)$ is the Fermi potential of the silicon body ( $k_{B}$ is Boltzmann's constant, $T$ is the absolute temperature, and $n_{i}$ is the intrinsic carrier density of silicon). Then substituting Eqs. (34) and (35) into $\psi_{s, \text { min }} \leq 2 \Phi_{B}$ and $\psi_{b, \text { min }} \leq 2 \Phi_{B}$, respectively, we can have $\Phi_{s} \leq 2 \Phi_{B}-\zeta$ and $\Phi_{b} \leq 2 \Phi_{B}-\zeta$, where $\zeta$ is given by

$$
\begin{aligned}
& \zeta=\left(V_{b i}+\frac{1}{2} V_{D S}-2 \Phi_{B}\right) \operatorname{csch}^{2}\left(\frac{L}{2 \lambda}\right) \\
& +\sqrt{\left(V_{b i}-2 \Phi_{B}\right)\left(V_{b i}+V_{D S}-2 \Phi_{B}\right)} \cosh \left(\frac{L}{2 \lambda}\right) \operatorname{csch}^{2}\left(\frac{L}{2 \lambda}\right),
\end{aligned}
$$


which approaches zero as $L$ is much larger than $\lambda$.

Plugging Eqs. (14) and (15) into $\Phi_{s} \leq 2 \Phi_{B}-\zeta$ and $\Phi_{b} \leq 2 \Phi_{B}-\zeta$, we can derive finally $V_{T f}$ and $V_{T b}$ as

$$
\begin{aligned}
V_{T f}= & V_{F B f}+\frac{q N_{A} t_{o f} t_{s i}}{2 \varepsilon_{o x}}+\left(1+\frac{t_{o f}}{\eta t_{s i}+t_{o b}}\right)\left(2 \Phi_{B}-\zeta\right) \\
& -\frac{t_{o f}}{\eta t_{s i}+t_{o b}}\left(V_{B S}-V_{F B b}-\frac{q N_{A} t_{o b} t_{s i}}{2 \varepsilon_{o x}}\right), \\
V_{T b}= & V_{F B b}+\frac{q N_{A} t_{o b} t_{s i}}{2 \varepsilon_{o x}}+\left(1+\frac{t_{o b}}{\eta t_{s i}+t_{o f}}\right)\left(2 \Phi_{B}-\zeta\right) \\
& -\frac{t_{o b}}{\eta t_{s i}+t_{o f}}\left(V_{G S}-V_{F B f}-\frac{q N_{A} t_{o f} t_{s i}}{2 \varepsilon_{o x}}\right) .
\end{aligned}
$$

From Eq. (37), we have

$$
\begin{gathered}
\frac{\partial V_{T f}}{\partial L}=\left(1+\frac{t_{o f}}{\eta t_{s i}+t_{o b}}\right)\left|\frac{\partial \zeta}{\partial u}\right| \frac{1}{2 \lambda}, \\
\frac{\partial V_{T f}}{\partial w}=-\left(1+\frac{t_{o f}}{\eta t_{s i}+t_{o b}}\right)\left|\frac{\partial \zeta}{\partial u}\right| \frac{L \lambda}{2 \lambda_{z}^{3}} \frac{\partial \lambda_{z}}{\partial w},
\end{gathered}
$$

where $u=L /(2 \lambda)$ and

$$
\begin{aligned}
& \frac{\partial \zeta}{\partial u}=-2\left(V_{b i}+\frac{1}{2} V_{D S}-2 \Phi_{B}\right) \cosh u \operatorname{csch}^{3} u \\
& -\sqrt{\left(V_{b i}-2 \Phi_{B}\right)\left(V_{b i}+V_{D S}-2 \Phi_{B}\right)}\left(1+\cosh ^{2} u\right) \operatorname{csch}^{3} u .
\end{aligned}
$$

Similarly, $\partial V_{T b} / \partial L$ and $\partial V_{T b} / \partial w$ can be written by exchanging $t_{o f}$ with $t_{o b}$ in Eqs. (39) and (40). From Eqs. (37) and (38), we find that $\Delta V_{T f} \equiv V_{T f}-\left.V_{T f}\right|_{L=\infty}$ and $\Delta V_{T b} \equiv V_{T b}-\left.V_{T b}\right|_{L=\infty}$ are independent of $V_{B S}$ and $V_{G S}$, respectively. Also note that $V_{T f}$ and $V_{T b}$ for a considerably wide gate device $\left(\lambda_{z} \approx w / \pi>>\lambda_{x}\right.$, i.e., $\lambda \approx \lambda_{x}$ ) coincide with the results given in the 2-D analytical model [18].

Let us consider the case that $t_{s i}=w, t_{o f}=t_{o b}=t_{o w}$, and $V_{G S}^{\prime}=V_{B S}^{\prime}$. In this case, the device becomes a surrounding gate (gate all around: GAA) MOSFET with rectangular silicon pillar or a fin FET. Using Eqs. (18), (24), and (32), we get

$$
\tan \left(\frac{t_{s i}}{2 \sqrt{2} \lambda}\right)=\eta \cot \left(\frac{t_{o x}}{\sqrt{2} \lambda}\right)
$$

Approximating Eq. (42) as the following cubic equation of $\xi=\lambda^{2}$ :

$$
\xi^{3}-a_{1} \xi^{2}-a_{2} \xi-a_{3} \approx 0
$$

where $\tau=2 t_{o x} / t_{s i}, \quad a_{1}=\left(t_{s i} / 2 \sqrt{2}\right)^{2}\left(\tau / \eta+\tau^{2} / 3\right)$, $a_{2}=\left(t_{s i} / 2 \sqrt{2}\right)_{6}^{4}\left(\tau / 3 \eta+\tau^{4} / 45\right)$, and $a_{3}=\left(t_{s i} / 2 \sqrt{2}\right)^{6}\left(2 \tau / 15 \eta+2 \tau^{6} / 945\right)$, we can derive $\lambda$ as

$$
\lambda \approx \sqrt{\frac{a_{1}}{3}+\sqrt[3]{R+\sqrt{Q^{3}+R^{2}}}+\sqrt[3]{R-\sqrt{Q^{3}+R^{2}}}},
$$

where $Q=-\left(3 a_{2}+a_{1}^{2}\right) / 9$ and $R=\left(9 a_{1} a_{2}+2 a_{1}^{3}+27 a_{3}\right) / 54$. Let us assume that a surrounding gate MOSFET with rectangular pillar is somewhat similar to a cylindrical surrounding gate MOSFET with $r_{s} \approx t_{s i} / 2$. Then we can find that the derived $\lambda$ for a rectangular shaped surrounding gate MOSFET becomes very similar to the result given in [20] where $\lambda$ for a cylindrical surrounding gate MOSFET has been derived. Thus, we may check the validity of Eqs. (20), (21), and (24).

\section{SAMPLE COMPUTATION AND DISCUSSION}

According to the expressions derived in Sec. II, $\lambda$, $\psi_{s}(y, 0)$, and the front gate threshold voltage $V_{T f}$ are computed and plotted in Figs. 2-7. In these computations, the following constants are used: $k_{B} T / q=0.0259[\mathrm{~V}]$ $n_{i}=1.45 \times 10^{10}\left[\mathrm{~cm}^{-3}\right], \quad \varepsilon_{o x}=3.9 \times 8.85 \times 10^{-14}[\mathrm{~F} / \mathrm{cm}], \varepsilon_{s i}=$ $=11.8 \times 8.85 \times 10^{-14}[\mathrm{~F} / \mathrm{cm}], \quad V_{b i}=0.55+\Phi_{B}[V], \quad \phi_{m s f}=$ $\phi_{m s b}=-0.55-\Phi_{B}[V] \quad\left(n^{+}-\right.$poly silicon gates are assumed), and $Q_{s s f}=Q_{s s b}=6.4 \times 10^{-9}\left[\mathrm{C} / \mathrm{cm}^{2}\right]$. In order to compare the presented model results with the simulated results given in [9], the values of device parameters have been chosen to be the same values used in the models under comparison. Since the presented model implies that 3-D model for a mesa-isolated SOI MOSFET becomes to be very similar to 2-D model in case that $w$ is not very narrow, we have compared our simulation results with the results given in the 2-D model of [9]. Simulation results given in 3-D models of [14, 17] are not compared with the presented model since the results in $[14,17]$ seem to be very complicated and rather ambiguous. Solving Eqs. (16) and (32) by means of the Newton-Raphson method, we can determine $\lambda_{x}$ and $\lambda_{z}$ as the corresponding largest values. It is worth noting that $\lambda$ becomes smaller than $\lambda_{x}$ and decreases as 


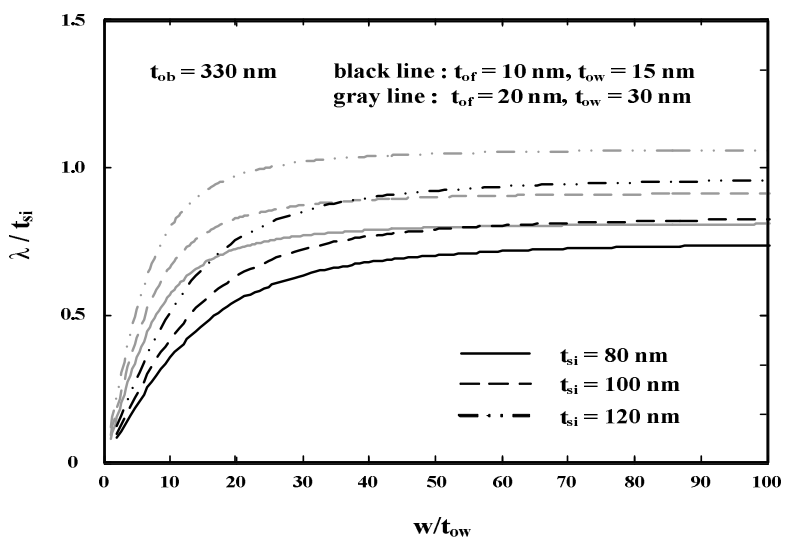

Fig. 2. Derived $\lambda / t_{s i}$ according to Eq. (24) is plotted as a function of $w / t_{o w}\left(t_{o b}=330 \mathrm{~nm}\right)$.

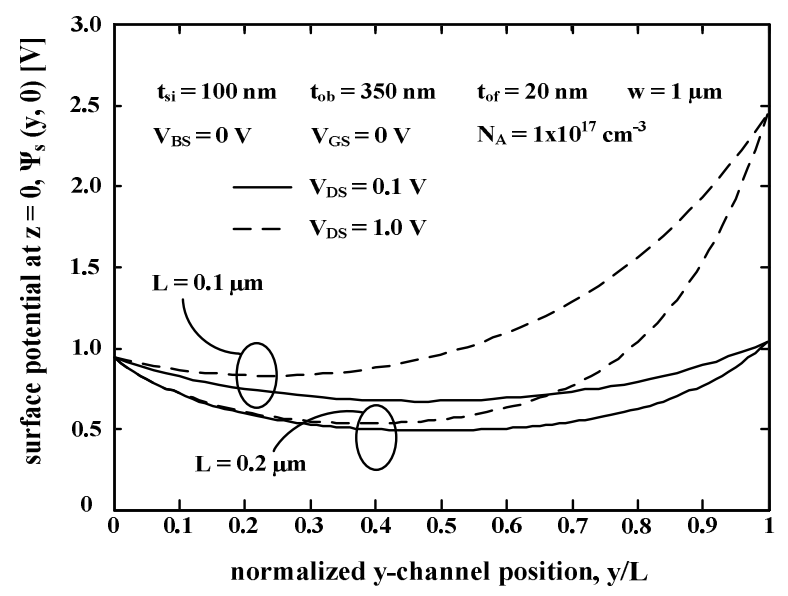

Fig. 3. Surface potential at $z=0$ versus $y / L$ $\left(w=1 \mu m, t_{s i}=100 \mathrm{~nm}, t_{o b}=350 \mathrm{~nm}, t_{o f}=20 \mathrm{~nm}, t_{o w}=30 \mathrm{~nm}\right.$, $\left.N_{A}=1 \times 10^{17} \mathrm{~cm}^{-3}, V_{B S}=0 \mathrm{~V}, V_{G S}=0 \mathrm{~V}\right)$.

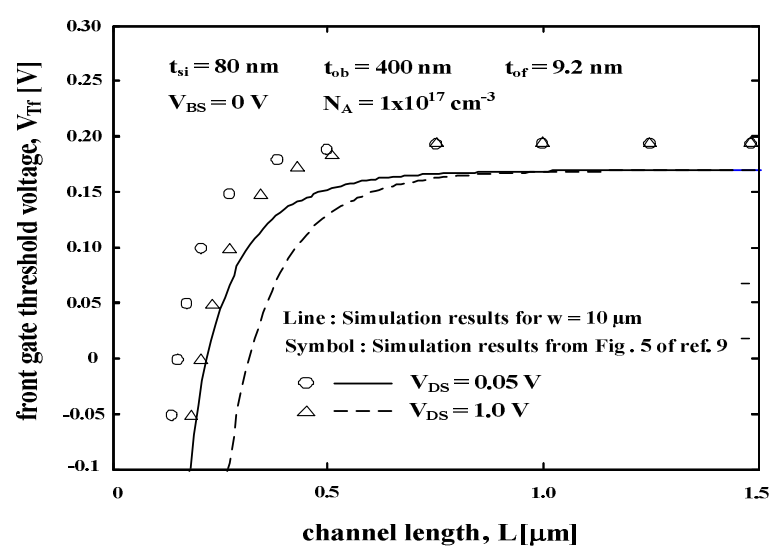

Fig. 4. Front gate threshold voltage versus channel length $\left(t_{s i}=80 \mathrm{~nm}, t_{o f}=9.2 \mathrm{~nm}, t_{o b}=400 \mathrm{~nm}, N_{A}=1 \times 10^{17} \mathrm{~cm}^{-3}\right.$, $\left.V_{B S}=0 \mathrm{~V}\right)$.

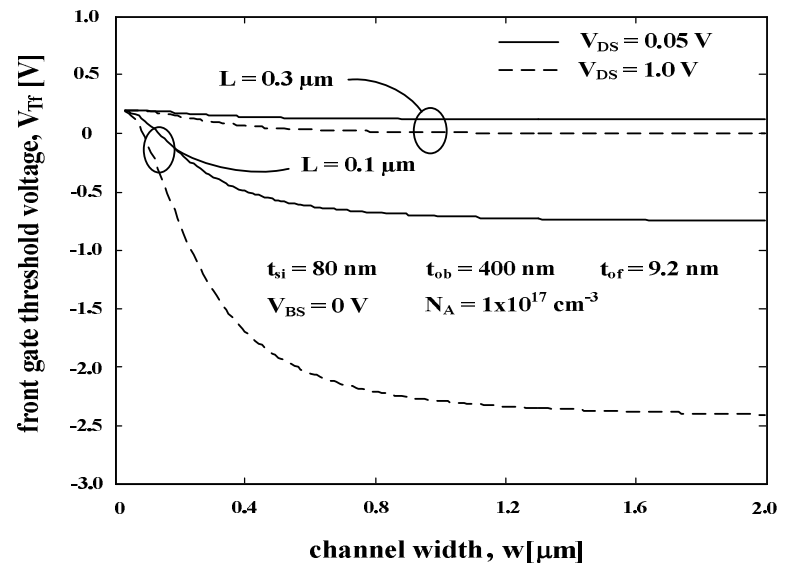

Fig. 5. Front gate threshold voltage versus channel width $\left(t_{s i}=80 \mathrm{~nm}, t_{o f}=9.2 \mathrm{~nm}, t_{o b}=400 \mathrm{~nm}, N_{A}=1 \times 10^{17} \mathrm{~cm}^{-3}\right.$, $\left.V_{B S}=0 \mathrm{~V}\right)$.

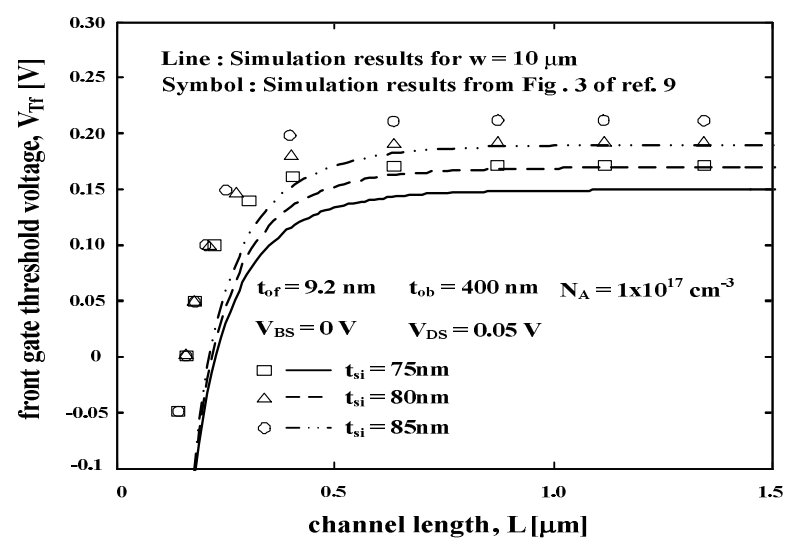

Fig. 6. Front gate threshold voltage versus channel length for various values of silicon film thickness $\left(t_{s i}=80 \mathrm{~nm}\right.$, $t_{o f}=9.2 \mathrm{~nm}, t_{o b}=400 \mathrm{~nm}, \quad N_{A}=1 \times 10^{17} \mathrm{~cm}^{-3}, \quad V_{B S}=0 \mathrm{~V}$, $\left.V_{D S}=0.05 \mathrm{~V}\right)$.

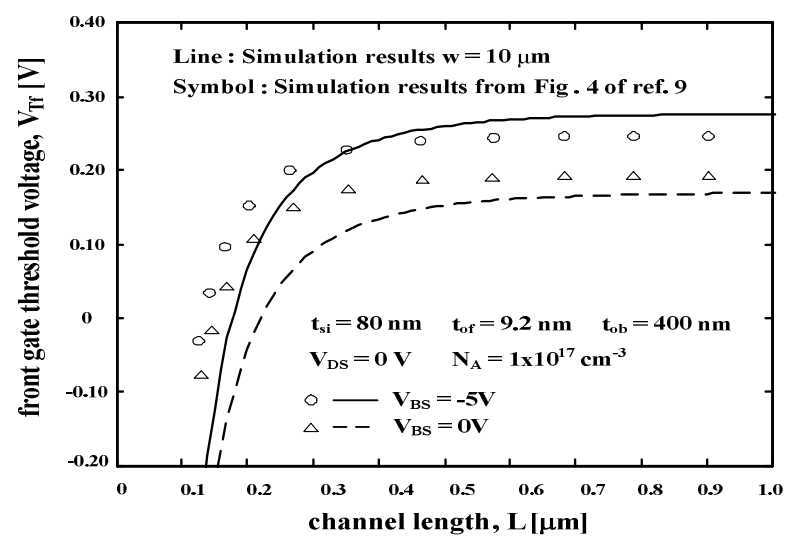

Fig. 7. Front gate threshold voltage versus channel length for $V_{B S}=0 \mathrm{~V} \quad$ and $\quad V_{B S}=-5 \mathrm{~V} \quad\left(t_{s i}=80 \mathrm{~nm}, \quad t_{o f}=9.2 \mathrm{~nm}\right.$, $\left.t_{o b}=400 \mathrm{~nm}, N_{A}=1 \times 10^{17} \mathrm{~cm}^{-3}, \quad V_{B S}=0 \mathrm{~V}, V_{D S}=0 \mathrm{~V}\right)$. 
channel width (front gate width) is decreased. In Fig. 2, the derived $\lambda / t_{s i}$ for various values of the silicon film thickness with $t_{o b}=330 \mathrm{~nm}$ is plotted as a function of $w / t_{o w}$. The $y$-variation of $\psi_{s}(y, 0)$ of a device with $L=0.1 \mu \mathrm{m}$ for $V_{D S}=0.1 \mathrm{~V}$ and $V_{D S}=1 \mathrm{~V}$ is plotted in Fig. 3. Also in Fig. 3, the derived $y$-variation of $\psi_{s}(y, 0)$ of a device with $L=0.2 \mu m$ for $V_{D S}=0.1 \mathrm{~V}$ and $V_{D S}=1 \mathrm{~V}$ is plotted. The derived dependency of the front gate threshold voltage $V_{T f}$ on the channel length for $V_{D S}=0.05 \mathrm{~V}$ and $V_{D S}=1 \mathrm{~V}$ is plotted in Fig. 4, where the drain-induced threshold voltage roll-off is shown evidently. Derived dependency of the front gate threshold voltage on the channel width for $V_{D S}=0.05 \mathrm{~V}$ and $V_{D S}=1 \mathrm{~V}$ is shown in Fig. 5. Derived dependencies of the front gate threshold voltage on the channel length for various values of silicon body thickness and back gate voltage are shown in Fig. 6 and 7, respectively. The simulation results from the presented model show a fairly good agreement with the results from [9], even though very small discrepancy about $\Delta V_{T} \approx 0.02 \mathrm{~V}$ is also found. Since this discrepancy is almost constant within the wide range of channel length, this would have resulted from the flat-band voltage shift. In the presented model, the potentials can be derived three-dimensionally as functions of infinite order of $x$ and $z$, while in the previously suggested 2-D model [18] the potentials were derived as functions of fifth-order of $x$. Considering that the drain-induced threshold voltage roll-off can be described accurately from a rigorous derivation of the front and back surface potentials, it can be expected that the presented model seems to derive the drain-induced threshold voltage roll-off fairly accurately.

\section{CONCLUSIONS}

As shown in Section II, starting from the potential expressions obtained by using the GCA and updating them iteratively by taking into account their $y$ - and $z$-variations, we can derive three-dimensionally the potentials in the silicon film (body), front, back, and side-wall oxide layers of a mesa-isolated fully depleted SOI MOSFET. From the derived three dimensional (3-D) potential expressions, we can derive the minimum values of the front and back surface potentials as closed-form expressions. These closed-form expressions seem to be much more convenient than the series expressions. Finally, using the reasonable physics-based threshold criterion, the front and back gate threshold voltages can be derived as the closed-form expressions in terms of various device parameters and applied bias voltages. It is worth noting that the presented model uses fewer assumptions and approximations in the analytical model procedure. Obtained results can be found to explain the drain-induced threshold voltage roll-off and the narrow width effect in a unified manner. Consequently, the threshold voltage expressions derived in the presented model can be used for estimating the front and back gate threshold voltages of a short channel fully depleted SOI MOSFET within a range of satisfactory accuracy.

\section{ACKNOWLEDGMENTS}

This work was supported by 2011 Hongik University Research Fund.

\section{REFERENCES}

[1] Lim H. Fossum J. R., "Threshold Voltage of ThinFilm Silicon-on-Insulator (SOI) MOSFET's", IEEE Trans. Electron Devices, vol. 30, no. 10, pp. 1244, 1983.

[2] Veeraghavan S. and Fossum J. G., "Short-channel effects in SOI MOSFET's", IEEE Trans. Electron Devices, vol. 36, no. 3, pp. 522, 1989.

[3] R. H. Yan, A. Ourmazd, K. F. Lee, "Scaling the Si MOSFET: from bulk to SOI to bulk," IEEE Trans. Electron Devices, vol. 39, no. 7, pp. 1704-1710, 1992.

[4] P. Francis, A. Terao, D. Flandre, F. Van de Wiele, "Modeling of ultrathin double-gate nMOS/SOI transistors," IEEE Trans. Electron Devices, vol. 41, no. 5, pp. 715-720, 1994.

[5] T. C. Hsiao, J. C. S. Woo, "Subthreshold characteristics of fully depleted submicrometer SOI MOSFET's," IEEE Trans. Electron Devices, vol. 42, no. 6, pp. 1120-1125, 1995.

[6] K. K. Young, "Short-channel effects in fully depleted SOI MOSFET's," IEEE Trans. Electron Devices, vol. 36, no. 2, pp. 399-402, 1989.

[7] J. C. S. Woo, K. W. Terrill, P. K. Vasudev, "Twodimensional analytical modeling of very thin SOI MOSFET's," IEEE Trans. Electron Devices, vol. 37, no. 9, pp. 1999-2000, 1990.

[8] Joachim H., Yamaguchi Y., Ishikawa K., Inoue Y., and Nishimura T., "Simulation Two-Dimensional analytical modeling of sub-threshold slope in ultrathin-film SOI MOSFET's down to $0.1 \mu \mathrm{m}$ gate length", IEEE Trans. Electron Devices, vol. 40, no. 
10, pp. 1812, 1993.

[9] S. R. Banna, P. C. H. Chan, P. K. Ko, C. T. Nguyen, and M. Chan, "Threshold Voltage Model for DeepSubmicrometer Fully Depleted SOI MOSFET's," IEEE Trans. Electron Devices, vol. 42, no. 11, pp. 1949-1955, 1995.

[10] J. Y. Guo, C. Y. Wu, "A new 2D analytical threshold-voltage model for fully depleted shortchannel SOI MOSFET's," IEEE Trans. Electron Devices, vol. 40, no. 9, pp. 1653-1661, 1993.

[11] K. Suzuki, Y. Tosaka, T. Sugii, "Analytical threshold voltage model for short channel doublegate MOSFET's," IEEE Trans. Electron Devices, vol. 43, no. 7, pp. 1166-1168, 1996.

[12] R. Zhang, K. Roy, "Low-Power high-performance Double-Gate Fully Depleted SOI Circuit Design," IEEE Trans. Electron Devices, vol. 49, no. 5, pp. 852-862, 2002.

[13] K. M. Chang, H. P. Wang, "A simple 2D analytical threshold voltage model for fully depleted shortchannel silicon-on-insulator MOSFET's," Semi-con Sci. Tech, vol. 19, no. 12, pp. 1397-1405, 2004.

[14] G. Katti, N. DasGupta, and A. DasGupta, "Threshold Voltage Model for Mesa-Isolated Small Geometry Fully Depleted SOI MOSFETs Based on Analytical Solution of 3-D Poisson's Equation," IEEE Trans. Electron Devices, vol. 51, no. 7, pp. 1169-1177, 2004.

[15] Wenwei Yang, Zhiping Yu, Lilin Tian, "Scaling Theory for FinFETs Based on 3-D Effects Investigation", IEEE Trans. Electron Devices, vol. 54, no. 5, 2007.

[16] Romain Ritzenthaler, Francois Lime, Olivier Faynot, Sorin Cristoloveanu, Benjamin Ininguez, "3D analytical modeling of subthreshold characteristics in vertical Multiple-gate FinFET transistors", Solid-State Electronics, 2011.

[17] Krishna Meel, R. Gopal, Deepak Bhatnagar, "Three-dimensional analytical modelling of front and back gate threshold voltages small geometry Fully Depleted SOI MOSFETs," Solid-State Electronics, vol. 62, pp. 174-184, 2011.

[18] C. H. Suh, "A simple analytical model for the front and back gate threshold voltages of a fully-depleted asymmetric SOI MOSFET," Solid-State Electronics, vol. 52, pp. 1249-1255, 2008.

[19] C. H. Suh, "Analytical model for deriving the threshold voltage of a short gate SOI MESFET with vertically non-uniformly doped silicon film", IET Circuits, Devices, and Systems, vol. 4, Iss. 6, pp. 525-530, 2010.

[20] C. H. Suh, "Two-Dimensional Analytical Model for Deriving the Threshold Voltage of a Short Channel Fully Depleted Cylindrical/Surrounding Gate MOSFET", Journal of Semiconductor Technology and Science, vol. 11, no. 2, pp. 111120, 2011.

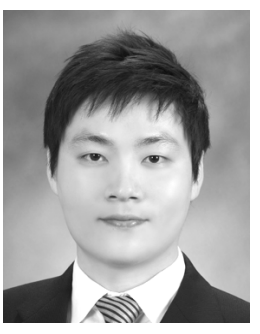

Jae Bin Lee was born in Seoul, Korea, in 1982. He received the B. S. degree from Hongik University in 2009, Department of Electronic and Electrical Engineering and the M. S. degree from Hongik University in 2011, Department of Electronic Engineering. In 2011 he joined the COMIZOA corp in Daejeon, Korea, and serving there as a circuit design Engineer. His areas of interest are physics of Power devices and integrated circuit.

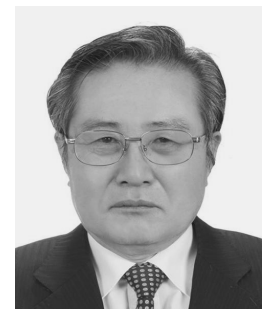

Chung Ha Suh was born in Daegu, Korea, in 1946. He received the B. S. degree from Seoul National University in 1969, Department of Electronic Engi- neering, the M. S. degree from Hongik University in 1975, and the Ph. D. degree in the area of semiconductor device physics from Seoul National University in 1981. In 1976 he joined the Department of Electronic Engineering, Hongik University, and served there as an Assistant Professor until 1978, and was promoted to a Full Professor in February, 1987. He was at the University of Minnesota from 1979 to 1980 as a visiting scholar and joined the Post Doc. program at the University of Pennsylvania from 1983 to 1984. At Hongik University, he served as Dean of Research Affairs and Dean of Graduate School of Industrial Information Technology from 1991 to 1999. His areas of interest are solid-state electronics and integrated circuit. He has published two text books in the area of physical electronics, and several papers in the IEEE TRANSACTIONS ON ELECTRON DEVICES, the Journal of Applied Physics, Solid-State Electronics, and other technical publications. Dr. Suh is a life-long member of the Korean Institute of Electronics Engineers and a member of IEEE. 